DESY 06-106

hep-ph/0607170

November, 2006

\title{
Inflaton Decay through Supergravity Effects
}

\author{
Motoi Endo ${ }^{1,2}$, Masahiro Kawasaki ${ }^{1}$, Fuminobu Takahashi ${ }^{1,2}$ and T. T. Yanagida ${ }^{3,4}$ \\ ${ }^{1}$ Institute for Cosmic Ray Research, University of Tokyo, \\ Chiba 277-8582, Japan \\ ${ }^{2}$ Deutsches Elektronen Synchrotron DESY, Notkestrasse 85, \\ 22607 Hamburg, Germany \\ ${ }^{3}$ Department of Physics, University of Tokyo, \\ Tokyo 113-0033, Japan \\ ${ }^{4}$ Research Center for the Early Universe, University of Tokyo, \\ Tokyo 113-0033, Japan
}

\begin{abstract}
We point out that supergravity effects enable the inflaton to decay into all matter fields, including the visible and the supersymmetry breaking sectors, once the inflaton acquires a non-vanishing vacuum expectation value. The new decay processes have great impacts on cosmology; the reheating temperature is bounded below; the gravitinos are produced by the inflaton decay in a broad class of the dynamical supersymmetry breaking models. We derive the bounds on the inflaton mass and the vacuum expectation value, which severely constrain high-scale inflations such as the hybrid and chaotic inflation models.
\end{abstract}




\section{Introduction}

In recent articles [1], we pointed out that high-scale inflations such as hybrid inflation models [2] are disfavored, since too many gravitinos are produced in reheating processes after the inflation if the hidden-sector field $z$ for supersymmetry (SUSY) breaking is completely neutral [3, 4, 5, 6]. However if the $z$ has charges of some symmetries like in gauge- and anomaly-mediation models [7, 8], dangerous operators are suppressed [5, 6, 1]

and there is no gravitino-overproduction problem\#1. Furthermore, such symmetries may suppress linear terms of the field $z$ in Kähler potential to avoid the Polonyi problem [9].

In this letter, we show that, as long as kinematically allowed, the inflaton $\phi$ decays into all matter fields that appear in the superpotential due to the supergravity (SUGRA) effect, once the inflaton acquires a non-vanishing vacuum expectation value (VEV), $\langle\phi\rangle$. For a typical inflaton mass in high-scale inflations, the inflaton may decay into the SUSY breaking sector fields, producing the $z$ field and the gravitinos in a broad class of the dynamical SUSY breaking (DSB) models [10, 11, 12, 13, 14, 15]. Since the decay proceeds independently of the charge of the $z$, the gravitino production from the inflaton decay is a generic problem, which is present even in the gauge- and anomaly-mediation models. In particular, the gravitinos are produced even if the Kähler potential is minimal. Further, the inflaton with a nonzero VEV can decay into the visible sector fields through the top Yukawa coupling, even if there is no direct interaction in the global SUSY limit. This enables us to set a lower limit on the reheating temperature of the inflation models. We derive constraints on the inflaton mass and the VEV, taking account of those new decay processes.

\section{Decay Processes}

The inflaton field $\phi$ couples to all matter fields due to the SUGRA effects, assuming a non-vanishing VEV. The relevant interactions with fermions are represented in terms of

\footnotetext{
${ }^{\# 1}$ See however Ref. [6] for other potential problems due to non-renormalizable couplings between the inflaton and the $z$.
} 
the total Kähler potential $G=K+\ln |W|^{2}$ in the Planck unit $M_{P}=1$ :

$$
\mathcal{L}=-\frac{1}{2} e^{G / 2} G_{\phi i j k} \phi \psi^{i} \psi^{j} \varphi^{k}+\text { h.c. }
$$

where $\varphi^{i}$ denotes a scalar field and $\psi^{i}$ is a fermion in 2-spinor representation. We assume $G_{i} \ll O(1)$ for all the fields other than the SUSY breaking field. The contribution proportional to $G_{\phi}$ may be suppressed in the inflaton decay because of the interference with the SUSY breaking field [5, 6].

In this section, we assume the minimal Kähler potential for simplicity. Then, there is no non-renormalizable term in the Kähler potential. Even when the inflaton field is secluded from the other fields in the global SUSY Lagrangian, the SUGRA corrections make its decay possible. At the vacuum, the coupling constants are expanded as

$$
G_{\phi i j k}=-\frac{W_{\phi}}{W} \frac{W_{i j k}}{W}+\frac{W_{\phi i j k}}{W} \simeq K_{\phi} \frac{W_{i j k}}{W}+\frac{W_{\phi i j k}}{W},
$$

where we assumed that the VEVs are negligibly small for all the fields other than the inflaton, and used $G_{\phi} \ll\langle\phi\rangle$ in the last equality. We find that the result is obviously invariant under the Kähler transformation, and these constants approach to zero in the global SUSY limit. Then the decay rates are evaluated as \#2

$$
\Gamma\left(\phi \rightarrow \psi^{i} \psi^{j} \varphi^{k}\right) \simeq \frac{N_{f}}{1536 \pi^{3}}\left|Y_{i j k}\right|^{2}\left(\frac{\langle\phi\rangle}{M_{P}}\right)^{2} \frac{m_{\phi}^{3}}{M_{P}^{2}},
$$

where $N_{f}$ is a number of the final state, and the Yukawa coupling $Y_{i j k} \equiv W_{i j k}$. Here we have neglected the masses of the final state particles, and used $K=\phi^{\dagger} \phi$ for the inflaton. We have also assumed that $\psi^{i}$ and $\psi^{j}$ are not identical particles. It is stressed again that the inflaton decay proceeds even when there is no direct interactions with the matter fields in the global SUSY limit.

The decay rates of the inflaton into the scalar particles become the same as the above results. In fact, with the scalar potential, $V=e^{G}\left(G^{i} G_{i}-3\right)$, the decay amplitude of $\phi^{*} \rightarrow \varphi^{i} \varphi^{j} \varphi^{k}$ is estimated as $V_{\bar{\phi} i j k}$. Since the inflaton has a large SUSY mass, the amplitude is approximately proportional to $e^{G / 2} G_{\phi i j k}$ multiplied by the inflaton mass, $m_{\phi} \simeq e^{G / 2}\left|G_{\bar{\phi}}^{\phi}\right|$. Therefore the decay rate satisfies $\Gamma\left(\phi^{*} \rightarrow \varphi^{i} \varphi^{j} \varphi^{k}\right) \simeq 3 \Gamma\left(\phi \rightarrow \psi^{i} \psi^{j} \varphi^{k}\right)$.

\footnotetext{
\#2 Similarly we obtain the 2-body decays, though the decay rate is suppressed by $O\left(10^{2}\right) \times M_{i j}^{2} / m_{\phi}^{2}$ with $M_{i j} \equiv W_{i j}$ compared to the 3-body decay rate with $Y=O(1)$. See also Ref. [16].
} 


\subsection{Lower bound on the reheating temperature}

Let us consider the inflaton decay through the top Yukawa coupling:

$$
W=Y_{t} T Q H_{u}
$$

where $Y_{t}$ is the top Yukawa coupling, and $T, Q$, and $H_{u}$ are the chiral supermultiplets of the right-handed top quark and left-handed quark doublet of the third generation, and up-type Higgs, respectively. The partial decay rate of the inflaton through the top Yukawa coupling is

$$
\Gamma_{T}=\frac{3}{128 \pi^{3}}\left|Y_{t}\right|^{2}\left(\frac{\langle\phi\rangle}{M_{P}}\right)^{2} \frac{m_{\phi}^{3}}{M_{P}^{2}},
$$

which sets a lower bound on the reheating temperature, $T_{R}$. We define the reheating temperature as

$$
T_{R} \equiv\left(\frac{\pi^{2} g_{*}}{10}\right)^{-\frac{1}{4}} \sqrt{\Gamma_{\phi} M_{P}}
$$

where $g_{*}$ counts the relativistic degrees of freedom, and $\Gamma_{\phi}$ denotes the total decay rate of the inflaton. Using Eqs. (5) and (6), we obtain the lower bound on the reheating temperature,

$$
T_{R} \gtrsim 1.9 \times 10^{3} \mathrm{GeV}\left|Y_{t}\right|\left(\frac{g_{*}}{200}\right)^{-\frac{1}{4}}\left(\frac{\langle\phi\rangle}{10^{15} \mathrm{GeV}}\right)\left(\frac{m_{\phi}}{10^{12} \mathrm{GeV}}\right)^{\frac{3}{2}} .
$$

We show the contours of the lower limit on the reheating temperature in Fig. 1, together with the results of the hybrid and smooth hybrid inflation models [2, 17]. If the inflaton mass $m_{\phi}$ and the $\mathrm{VEV}\langle\phi\rangle$ are too large, the reheating temperature may exceed the upper bound due to the abundance of the gravitinos produced by particle scattering in the thermal plasma; the recent results are given in [18, 19, 20] for the unstable gravitino and in [21] for the stable one (see Ref. [1] for the summarized results). In particular, for the smooth hybrid inflation model, the reheating temperature is necessarily higher than $10^{6} \mathrm{GeV}$, which is difficult to be reconciled with the gravitino of $m_{3 / 2}=O(0.1-$ 1) $\mathrm{TeV}[18]$.

\subsection{Inflaton decay into the gravitinos}

The new decay process enables the inflaton to decay into the SUSY breaking sector. Through Yukawa interactions containing the SUSY breaking field, the gravitino is pro- 
duced from the inflaton decay. It should be noted that the gravitino can be produced even without non-renormalizable coupling $|\phi|^{2} z z$ in the Kähler potential. The existence of such coupling is crucial for the gravitino pair production [1, 6], if the inflaton mass is larger than the scalar mass of the SUSY breaking field $z$.

Let us consider the Yukawa interactions of the SUSY breaking sector fields \#3. From the point of view of naturalness, the DSB scenarios [10] are attractive. In a wide class of the DSB models [11, 12, 13, 14, 15], including ones reduced from $\mathcal{N}=2$ SUSY quiver gauge theories [22], there exist such Yukawa interactions at the quark level \#4. When one considers the inflaton decay into the SUSY breaking sector, the quark-level interactions should be used, instead of the one written in terms of the composite fields in the lowenergy effective theory, since the inflaton mass scale is larger than the strong-coupling scale. The fields in the SUSY breaking sector typically acquire masses of the DSB scale $\Lambda \sim \sqrt{m_{3 / 2} M_{P}}$, while the inflaton in the high-scale inflation models such as the hybrid inflation model tends to satisfy with $m_{\phi} \gtrsim \Lambda$. Then the inflaton can decay into the hidden sector fields.

The SUSY breaking field in the low-energy effective theory is either an elementary or composite field. Let us denote the SUSY breaking field by $z$ throughout this letter. If the $z$ is an elementary field and appears in a Yukawa interaction, its fermionic component is directly produced by the inflaton decay. On the other hand, if $z$ is composite, the produced hidden quarks will form QCD-like jets, ending up with the hidden mesons and/or baryons, together with (possibly many) z fields. In both cases, since the fermionic component of $z$ is identified with the goldstino, the gravitino is produced by the inflaton decay. In addition, the scalar component of $z$ and the other SUSY breaking sector fields may also decay into the gravitino.

\#3 If there exists a linear term $W=\mu^{2} z$ in the superpotential, the inflaton $\phi$ mixes with $z$ in the vacuum. Even if the minimum of $z$ during inflation coincides with that after inflation, the coherent oscillations of $z$ field is induced by the inflaton via the mixing [6]. However, the induced amplitude of $z$ is so small that it is cosmologically harmless.

\#4 Note, however, that those models without Yukawa interactions can also break the SUSY dynamically. Examples include $\mathrm{SU}(5)$ model with $\mathbf{5}^{*}$ and 10, and $\mathrm{SO}(10)$ with 16 [23, [1]. Then the constraint due to the gravitino production discussed in this letter is not applied for such models. 
Therefore the gravitino production rate is expressed as \#5

$$
\Gamma_{3 / 2}=\frac{C}{1536 \pi^{3}}\left(\frac{\langle\phi\rangle}{M_{P}}\right)^{2} \frac{m_{\phi}^{3}}{M_{P}^{2}}
$$

where $C$ is determined by the decay processes; the degrees of freedom of the decay products, the decay chains, the coupling constants of the Yukawa interactions in the SUSY breaking sector, and form factors of the hidden mesons and/or baryons. Although the constant $C$ strongly depends on the models, unless all the Yukawa couplings are extremely suppressed, $C$ is expected to be $O(1)$ or larger. The gravitino abundance is then given by \#6

$$
Y_{3 / 2}=8 \times 10^{-14} C\left(\frac{g_{*}}{200}\right)^{-\frac{1}{2}}\left(\frac{T_{R}}{10^{6} \mathrm{GeV}}\right)^{-1}\left(\frac{m_{\phi}}{10^{12} \mathrm{GeV}}\right)^{2}\left(\frac{\langle\phi\rangle}{10^{15} \mathrm{GeV}}\right)^{2},
$$

for $m_{\phi} \gtrsim \Lambda$.

For the inflaton mass, $m_{\phi} \lesssim \Lambda$, the inflaton decay into the SUSY breaking sector is likely to be kinematically forbidden. However, the gravitino pair production from the inflaton decay instead becomes important [1, 5, 6], if the inflaton mass is smaller than the mass of the scalar component of the $z$ field. We here assume that the mass of the $z$ field is $O(\Lambda)$ in the DSB scenarios. The gravitino pair production rate is given by [1, 3, 6 ]

$$
\Gamma_{3 / 2}^{(\text {pair })}=\frac{3}{288 \pi}\left(\frac{\langle\phi\rangle}{M_{P}}\right)^{2} \frac{m_{\phi}^{3}}{M_{P}^{2}}
$$

which is larger than Eq. (8) by two orders of magnitude. Note that, as long as $m_{\phi} \lesssim \Lambda$, the inflaton decay into a pair of the gravitinos occurs at the rate shown above, even if the Kähler potential is minimal. The gravitino abundance is then given by

$$
Y_{3 / 2}=2 \times 10^{-11}\left(\frac{g_{*}}{200}\right)^{-\frac{1}{2}}\left(\frac{T_{R}}{10^{6} \mathrm{GeV}}\right)^{-1}\left(\frac{m_{\phi}}{10^{12} \mathrm{GeV}}\right)^{2}\left(\frac{\langle\phi\rangle}{10^{15} \mathrm{GeV}}\right)^{2},
$$

for $m_{\phi} \lesssim \Lambda$.

There are tight constraints on the gravitino abundance from BBN if the gravitino is unstable, and from the dark matter (DM) abundance for the stable gravitino. In

\footnotetext{
\#5 The same result can be obtained from the SUGRA Lagrangian. Actually, assuming the Yukawa coupling $W=z Q Q$, the decay rate of $\phi \rightarrow \tilde{G} Q Q$ in the goldstino picture is reproduced by evaluating the $Q$-mediating diagram in the unitary gauge, where $\tilde{G}$ is the goldstino.

\#6 We have assumed here that there is no entropy production 24] after the reheating.
} 
particular, for the gravitinos produced by thermal scatterings, the gravitino abundance is related to $T_{R}$ by using [25, 18]

$$
\begin{aligned}
Y_{3 / 2}^{(t h)} & \simeq 1.9 \times 10^{-12}\left[1+\left(\frac{m_{\tilde{g}_{3}}^{2}}{3 m_{3 / 2}^{2}}\right)\right]\left(\frac{T_{\mathrm{R}}}{10^{10} \mathrm{GeV}}\right) \\
& \times\left[1+0.045 \ln \left(\frac{T_{\mathrm{R}}}{10^{10} \mathrm{GeV}}\right)\right]\left[1-0.028 \ln \left(\frac{T_{\mathrm{R}}}{10^{10} \mathrm{GeV}},\right)\right],
\end{aligned}
$$

where we have taken $N=3$ for QCD and $m_{\tilde{g}_{3}}$ is the gluino mass evaluated at $T=T_{R}$. Since the gravitino abundance $Y_{3 / 2}^{(t h)}$ is roughly proportional to $T_{R}$, both are bounded above. Substituting the upper bounds into Eqs. (9) and (11), we obtain constraints on the mass and the VEV of the inflaton.

Here we simply quote the bounds on $Y_{3 / 2}$ and $T_{R}$ summarized in Ref. [1], for representative values of the gravitino mass: $m_{3 / 2}=1 \mathrm{GeV}, 1 \mathrm{TeV}$ and $100 \mathrm{TeV}$. For the stable gravitino, the gravitino abundance should not exceed the DM abundance [21],

$$
m_{3 / 2} Y_{3 / 2} \leq \Omega_{\mathrm{DM}} \frac{\rho_{c}}{s} \lesssim 4.7 \times 10^{-10} \mathrm{GeV}
$$

where $\rho_{c}$ is the critical density, and we used $\Omega_{\mathrm{DM}} h^{2} \lesssim 0.13$ at $95 \%$ C.L. [26] in the second inequality. Here we have neglected the contribution from the decay of the next-to-lightest SUSY particle. The upper bound on $T_{R}$ can be obtained by substituting Eq. (13) into Eq. (12) as

$$
T_{R} \lesssim 3 \times 10^{7} \mathrm{GeV}\left(\frac{m_{\tilde{g}_{3}}}{500 \mathrm{GeV}}\right)^{-2}\left(\frac{m_{3 / 2}}{1 \mathrm{GeV}}\right)
$$

for $m_{3 / 2} \simeq 10^{-4}-10 \mathrm{GeV}$. For the unstable gravitinos with $m_{3 / 2}=1 \mathrm{TeV}$, the bounds come from BBN: [1]

$$
Y_{3 / 2} \lesssim\left\{\begin{array}{l}
4 \times 10^{-17}, \quad\left(B_{h} \simeq 1\right), \\
3 \times 10^{-14}, \quad\left(B_{h} \simeq 10^{-3}\right),
\end{array}\right.
$$

where $B_{h}$ denotes the hadronic branching ratio of the gravitino. The bounds on $T_{R}$ are given by

$$
T_{R} \lesssim\left\{\begin{array}{lc}
3 \times 10^{5} \mathrm{GeV}, & \left(B_{h} \simeq 1\right), \\
2 \times 10^{8} \mathrm{GeV}, & \left(B_{h} \simeq 10^{-3}\right),
\end{array}\right.
$$

For the unstable gravitino with $m_{3 / 2}=100 \mathrm{TeV}$, the constraint comes from the abundance of the lightest SUSY particle (LSP) produced by the gravitino decay. In the anomalymediated SUSY breaking models with the wino LSP, one LSP remains as a result of the 
decay of one gravitino, since the gravitino decay temperature is rather low. The bounds read

$$
\begin{aligned}
Y_{3 / 2} & \lesssim 2 \times 10^{-12}\left(\frac{m_{3 / 2}}{100 \mathrm{TeV}}\right)^{-1} \\
T_{R} & \lesssim 9 \times 10^{9}\left(\frac{m_{3 / 2}}{100 \mathrm{TeV}}\right)^{-1} \mathrm{GeV}
\end{aligned}
$$

where we have used the relation,

$$
m_{\tilde{W}}=\frac{\beta_{2}}{g_{2}} m_{3 / 2} \simeq 2.7 \times 10^{-3} m_{3 / 2}
$$

where $\beta_{2}$ and $g_{2}$ are the beta function and the gauge coupling of $S U(2)_{L}$.

We show the bounds on the mass and the VEV of the inflaton, obtained by substituting the above upper bounds on $Y_{3 / 2}$ and $T_{R}$ into Eqs. (9) and (11), in Figs. 2, 3 and 4, The bounds are severer than that due to the lower bound on $T_{R}$ shown in Fig. 1, and a significant fraction of the parameter space is excluded by the gravitino production. The bound shown in Fig. 2 does not change for the stable gravitinos with $m_{3 / 2} \simeq 100 \mathrm{keV}-$ $10 \mathrm{GeV}$, since both the upper bound on $T_{R}$ is proportional to $m_{3 / 2}$. It should be noted that the bounds become severer for lower $T_{R}$, since $T_{R}$ is set to be the largest allowed value.

\section{Constraints on Inflation Models}

To see the impacts of the decay processes discussed in the previous section, let us first consider the hybrid inflation model [2]. The hybrid inflation model contains two kinds of superfields: one is $\phi$ which plays a role of inflaton and the others are waterfall fields $\psi$ and $\tilde{\psi}$.

The superpotential $W(\phi, \psi, \tilde{\psi})$ for the hybrid inflaton is

$$
W(\phi, \psi, \tilde{\psi})=\phi\left(\mu^{2}-\lambda \tilde{\psi} \psi\right)
$$

where $\mu$ determines the inflation energy scale, and $\psi$ and $\tilde{\psi}$ are assumed to be charged under $U(1)$ gauge symmetry. Here $\lambda$ is a coupling constant and $\mu$ is the inflation energy scale. The potential minimum is located at $\langle\phi\rangle=0$ and $\langle\psi\rangle=\langle\tilde{\psi}\rangle=\mu / \sqrt{\lambda}$ in the SUSY 


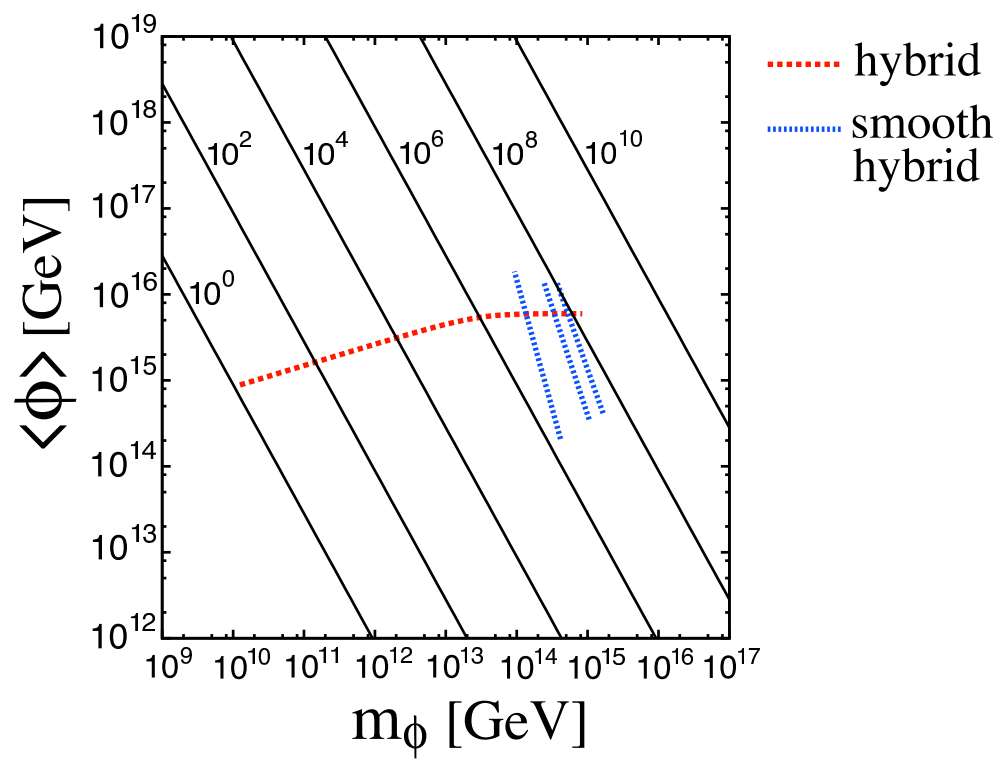

Figure 1: Contours of the lower bound on the reheating temperature $T_{R}$, denoted by the solid lines. We set $g_{*}=228.75$ and $Y_{t}=0.6$. The typical values of $\langle\phi\rangle$ and $m_{\phi}$ for the hybrid and smooth hybrid inflation models ( $n=2,3$ and 4 from left to right) are also shown.

limit. For a successful inflation \#7, $\mu$ and $\lambda$ are related as

$$
\begin{aligned}
& \mu \simeq 2 \times 10^{-3} \lambda^{1 / 2} \text { for } \lambda \gtrsim 10^{-3}, \\
& \mu \simeq 2 \times 10^{-2} \lambda^{5 / 6} \text { for } \lambda \lesssim 10^{-3},
\end{aligned}
$$

where $\lambda$ varies from $10^{-5}$ to $10^{-1}[27] \# 8$.

After inflation ends, the universe is dominated by both the inflaton $\phi$ and a combination of the waterfall fields, $\psi_{+} \equiv(\psi+\tilde{\psi}) / \sqrt{2}$, while the other combination, $\psi_{-} \equiv$ $(\psi-\tilde{\psi}) / \sqrt{2}$, gives negligible contribution to the total energy of the universe due to the $D$-flat condition. The inflaton $\phi$ almost maximally mixes with $\psi_{+}$to form the mass eigenstates [1],

$$
\phi_{ \pm} \equiv \frac{\phi \pm \psi_{+}}{\sqrt{2}} .
$$

\#7 We require the right magnitude of the density fluctuations and the spectral index less than or equal to unity [26]. The e-folding number is set to be $N_{e}=50$.

\#8 Note that, in this type of hybrid inflation, there exists a problem of cosmic string formation because $\psi$ and $\tilde{\psi}$ have $U(1)$ gauge charges. To avoid the problem the coupling $\lambda$ should be small as, $\lambda \lesssim 10^{-4}[28$. Here we do not take this problem seriously, since the cosmic strings are not produced if the gauge group is extended to a non-Abelian group [29, 30]. 


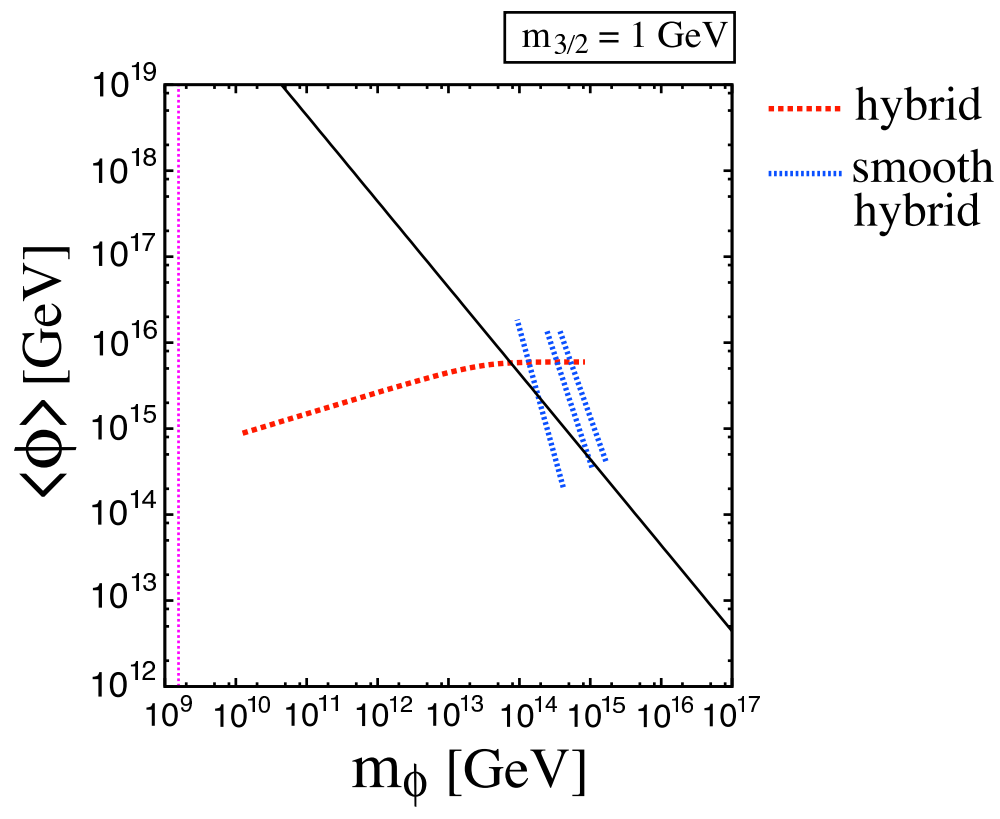

Figure 2: Constraints from the abundance of the gravitino produced from the inflaton decay, for $m_{3 / 2}=1 \mathrm{GeV}$. We set $g_{*}=228.75$ and $C=1$. The region above the solid line is excluded. $T_{R}$ is set to be the largest allowed value, and the bound becomes severer for lower $T_{R}$. The vertical dotted line corresponds to $m_{\phi}=\sqrt{m_{3 / 2} M_{P}}$. For $m_{\phi} \lesssim \sqrt{m_{3 / 2} M_{P}}$, the inflaton decay into the SUSY breaking sector is expected to be kinematically forbidden; however the gravitino pair production instead puts a severer constraint [3, 4, 5, 6]. The typical values of $\langle\phi\rangle$ and $m_{\phi}$ for the hybrid and smooth hybrid inflation models are also shown.

The VEVs and the masses of these mass eigenstates are given by

$$
\left\langle\phi_{ \pm}\right\rangle=\frac{\mu}{\sqrt{\lambda}}, \quad m_{\phi_{ \pm}}=\sqrt{2} \lambda\left\langle\phi_{ \pm}\right\rangle
$$

Since the two mass eigenstates $\phi_{ \pm}$have the equal decay rates due to the (almost) maximal mixing, we can simply treat the reheating process just like a single-field inflation model; the reheating is parameterized by a single parameter, $T_{R}$ (or equivalently, $\Gamma_{\phi}$ ) \#9.

We have plotted the mass and the VEV given by Eq. (22) for $\lambda=10^{-5}-10^{-1}$ in Figs. 1, 2, 3, and 4. From Fig. 1, one can see that the lower bound on the reheating temperature

\#9 The preheating [31, 32, 33. is known to occur in this model, and if it occurs, the homogeneous mode of the inflaton and the waterfall fields disappear soon and the excited particles are produced. This instability itself does not affect our discussion, since these excited particles will decay perturbatively into the lighter fields in the end. 


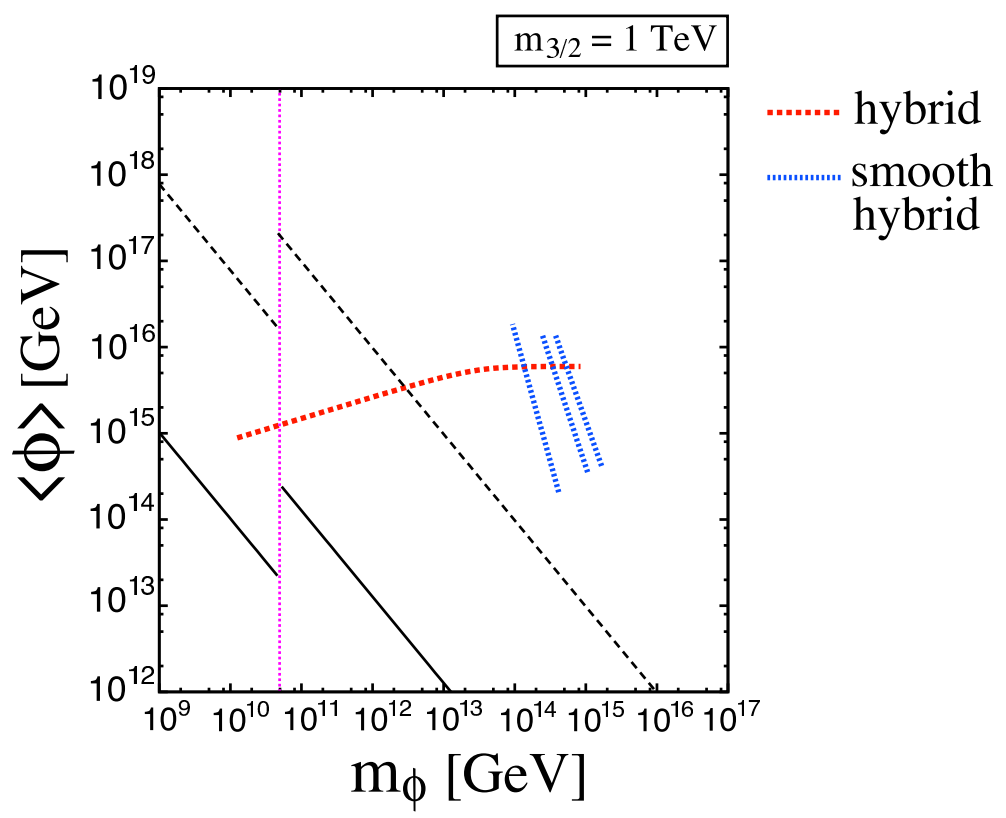

Figure 3: Same as Fig. 2 but for $m_{3 / 2}=1 \mathrm{TeV}$. The solid and dashed lines are for the hadronic branching ratio $B_{h}=1$ and $10^{-3}$, respectively.

broadly ranges from $O(1) \mathrm{GeV}$ up to $O\left(10^{8}\right) \mathrm{GeV}$. The gravitino problem excludes some fraction of the parameter space; for instance, $m_{\phi_{ \pm}} \gtrsim O\left(10^{13}\right) \mathrm{GeV}$ is excluded for $m_{3 / 2}=$ $1 \mathrm{TeV}$ with $B_{h}=1$, since the reheating temperature exceeds $10^{6} \mathrm{GeV}$ (see Eq. (15)). The gravitino production from the inflaton decay actually gives severer bounds as shown in Figs. 2, 3, and 4. In particular, the entire parameter region is excluded for $m_{3 / 2}=1 \mathrm{TeV}$ with $B_{h}=1$. For much larger or smaller $m_{3 / 2}$, the constraints on the hybrid inflation model is relaxed, and the region with relatively small $m_{\phi}$ is allowed. Note, however, that it then tends to be disfavored by WMAP three year data [26] since the predicted spectral index $n_{s}$ approaches to unity \#10. In other words, $n_{s} \simeq 1$ is necessary for the hybrid inflation model to be compatible with the gravitino overproduction from the inflaton decay.

Next let us consider a smooth hybrid inflation model [17]. The superpotential of the

\#10 Note that the hybrid inflation produces negligible tensor fluctuations and hence we should take the WMAP constraint on $n_{s}$ for no tensor mode, $n_{s}=0.95 \pm 0.02$. 


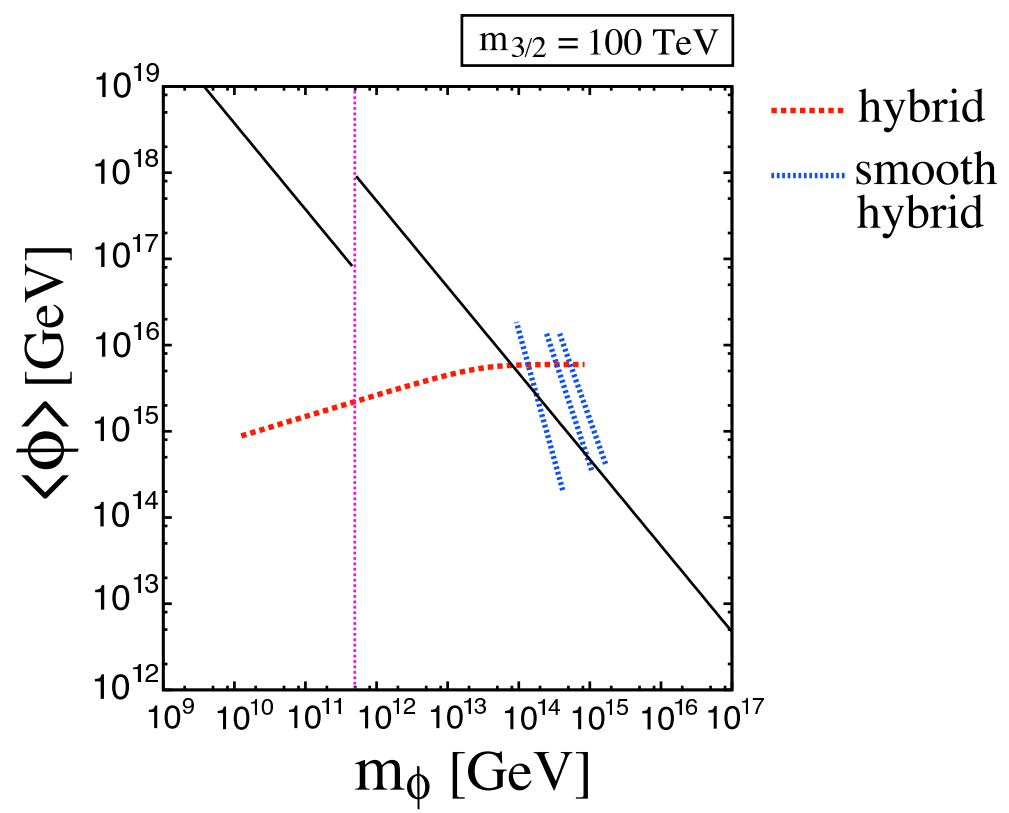

Figure 4: Same as Fig. 2 but for $m_{3 / 2}=100 \mathrm{TeV}$.

inflaton sector is

$$
W(\phi, \psi, \tilde{\psi})=\phi\left(\mu^{2}-\frac{(\tilde{\psi} \psi)^{n}}{M^{2 n-2}}\right),
$$

where $M$ is an effective cut-off scale, and $n \geq 2$ is an integer. The vacuum of the scalar potential is located at $\langle\phi\rangle=0$ and $\langle\psi\rangle=\langle\tilde{\psi}\rangle=\left(\mu M^{n-1}\right)^{1 / n}$ in the global SUSY limit. Note that $\psi=\tilde{\psi}$ always holds due to the additional $D$-term potential. As in the hybrid inflation model, one of the combination, $\psi^{(+)} \equiv(\psi+\tilde{\psi}) / \sqrt{2}$, almost maximally mixes with $\phi$ to form the mass eigenstates $\phi_{ \pm}$defined by Eq. (21). The VEVs and masses of $\phi_{ \pm}$ are given by

$$
\left\langle\phi_{ \pm}\right\rangle=\left(\mu M^{n-1}\right)^{1 / n}, \quad m_{\phi_{ \pm}}=\sqrt{2} n \mu^{2} /\langle\psi\rangle,
$$

which are plotted in Figs. 1, 2, 3, and 4, in the case of $n=2,3$ and 4. The ranges of the parameters are determined by requiring both a successful inflation with a large enough e-folding number and the validity of the effective description Eq. (23). The scalar spectral index is then predicted to be $n_{s} \simeq 0.97$, which is slightly smaller than the simple hybrid inflation model. From Fig. 1, we can see that the smooth hybrid inflation model is incompatible with the gravitino of $m_{3 / 2}=1 \mathrm{TeV}$, due to the too high reheating 
temperature. Further, taking account of the gravitino production from the inflaton decay, the smooth hybrid inflation model gets in more trouble for a broad range of the gravitino mass (see Figs. 2, 3, and 4). Note also that the constraints get severer for larger $n$.

Lastly, let us mention that those problems stated above can be avoided in the chaotic inflation model with a $Z_{2}$ symmetry. A chaotic inflation [34] is realized in SUGRA, based on a Nambu-Goldstone-like shift symmetry of the inflaton chiral multiplet $\phi$ [35]. Namely, we assume that the Kähler potential $K\left(\phi, \phi^{\dagger}\right)$ is invariant under the shift of $\phi$,

$$
\phi \rightarrow \phi+i A
$$

where $A$ is a dimensionless real parameter. Thus, the Kähler potential is a function of $\phi+\phi^{\dagger} ; K\left(\phi, \phi^{\dagger}\right)=K\left(\phi+\phi^{\dagger}\right)=c\left(\phi+\phi^{\dagger}\right)+\frac{1}{2}\left(\phi+\phi^{\dagger}\right)^{2}+\cdots$, where $c$ is a real constant and must be smaller than $O(1)$ for a successful inflation. We will identify its imaginary part with the inflaton field $\varphi \equiv \sqrt{2} \operatorname{Im}[\phi]$. Moreover, we introduce a small breaking term of the shift symmetry in the superpotential in order for the inflaton $\varphi$ to have a potential:

$$
W(\phi, \psi)=m \phi \psi
$$

where we introduced a new chiral multiplet $\psi$, and $m \simeq 10^{13} \mathrm{GeV}$ determines the inflaton mass. The scalar potential is given by

$$
V(\eta, \varphi, \psi) \simeq \frac{1}{2} m^{2} \varphi^{2}+m^{2}|\psi|^{2}
$$

where we set the real part of $\phi$ to be at the vacuum. For $\varphi \gg 1$ and $|\psi|<1$, the $\varphi$ field dominates the potential and the chaotic inflation takes place (for details see Refs [35]).

Although $\varphi$ does not acquire any finite VEV, the linear term in the Kähler potential behaves exactly the same as a VEV. Therefore, the decay rates given by Eqs. (5) and (8) apply to the inflaton $\varphi$, if one replaces $\langle\phi\rangle$ with $c$, the coefficient of the linear term. If $c$ is sizable, the chaotic inflation model of this type as well may encounter the cosmological difficulties. However, one can suppress such linear term by assuming an approximate $Z_{2}$ symmetry. Therefore the problems mentioned above can be avoided in the chaotic inflation model. 


\section{Discussion and Conclusions}

So far we have focused on the inflaton decay into the visible sector and the gravitinos. Since the inflaton couples to all matter fields once it acquires a finite VEV, the inflaton can also decay into the hidden and/or messenger sector. The decay may cause another cosmological problem. For instance, if the messenger fields are produced by the inflaton decay, and if the lightest messenger particle is stable, the abundance of such stable particle may easily exceed the present DM abundance. The production of the hidden sector field may be faced with the similar problem. Therefore, the inflaton decay process shown in this letter can put a constraint on the structure of the hidden and/or messenger sector.

The SUSY breaking may occur at tree level as well, although we have assumed the DSB scenarios in the previous sections. Our discussion on the gravitino production from the inflaton decay actually applies to any SUSY breaking models containing Yukawa interactions with sizable couplings. For instance, the O'Raifeartaigh-type models [36] may contain such terms. Note that a linear term in the superpotential as in the Polonyi model only induces a small mixing with the inflaton.

It depends on the Yukawa coupling constants in the SUSY breaking sector how much the gravitinos are produced by the inflaton decay. Although we have assumed $C \gtrsim O(1)$ in the above discussion, $C$ may be smaller if all the couplings are extremely suppressed. If this is the case, the constraints shown in Figs. 2, 3] and 4 are relaxed. Note that, in this case, the gravitino production sets severe constraint on the SUSY breaking sector, instead of the inflaton parameters. We have assumed no entropy production late after the reheating of inflation throughout this letter. If a late-time entropy production occurs [24], the constraints derived in the previous sections can be relaxed. Another even manifest solution to the gravitino overproduction problem is to assume the gravitino mass $m_{3 / 2}<$ $O(10) \mathrm{eV}$ [37]. In this case, the produced gravitinos get into thermal equilibrium due to relatively strong interactions with the standard-model particles, and such light gravitinos are cosmologically harmless.

Since the inflaton with a nonzero VEV couples to all matter fields that appear in the superpotential, it also decays into the right-handed neutrinos through the large Majorana mass term. The non-thermally produced right-handed neutrinos may generate the baryon 
asymmetry of the universe through the leptogenesis [38, 39]. Since the abundance of the right-handed neutrino is generically correlated with that of the gravitino produced in a similar way, $C \ll 1$ is required for the unstable gravitinos to realize the successful leptogenesis. However, for the stable gravitinos, the non-thermal leptogenesis may work even if $C=O(1)$. This opens up an interesting way to induce the non-thermal leptogenesis; the right-handed neutrinos are produced by the inflaton decay even if there is no direct coupling in the global SUSY limit; they are produced simply because the Majorana mass is large (but smaller than the inflaton mass). Detailed discussion on this topic will be presented elsewhere.

In this letter, we have shown that, once the inflaton acquires a finite VEV, it can decay into all matter fields via the SUGRA effect, as long as kinematically allowed. It is a striking feature that the decay occurs even without the direct couplings in the global SUSY limit. The inflaton with a nonzero VEV can therefore decay into the visible sector fields through the top Yukawa coupling, which has enabled us to set a lower limit on the reheating temperature. For a typical inflaton mass in high-scale inflations such as the hybrid and chaotic inflation models, the inflaton can decay into the SUSY breaking sector fields, producing the gravitinos in a broad class of the DSB models. We have seen that the gravitino production from the inflaton decay severely constrains the highscale inflation models. We would like to stress again that the gravitino production from the inflaton decay is a generic problem; it is present even in the gauge- and anomalymediation models, since the decay proceeds irrespective of whether the SUSY breaking field $z$ is charged under some symmetries or not. In particular, the gravitinos are produced even if the Kähler potential is minimal. One of the solution is to assign a symmetry on the inflaton field to forbid a nonzero VEV and a linear term in the Kähler potential. In fact, the chaotic inflation model with an approximate $Z_{2}$ symmetry can avoid the problem.

\section{Acknowledgments}

M.E and F.T. would like to thank the Japan Society for Promotion of Science for financial support. The work of T.T.Y. has been supported in part by a Humboldt Research Award. 


\section{References}

[1] M. Kawasaki, F. Takahashi and T. T. Yanagida, Phys. Lett. B 638, 8 (2006); arXiv:hep-ph/0605297.

[2] E. J. Copeland, A. R. Liddle, D. H. Lyth, E. D. Stewart and D. Wands, Phys. Rev. D 49, 6410 (1994);

G. R. Dvali, Q. Shafi and R. K. Schaefer, Phys. Rev. Lett. 73, 1886 (1994);

A. D. Linde and A. Riotto, Phys. Rev. D 56, 1841 (1997).

[3] M. Endo, K. Hamaguchi and F. Takahashi, Phys. Rev. Lett. 96, 211301 (2006);

S. Nakamura and M. Yamaguchi, arXiv:hep-ph/0602081.

[4] T. Asaka, S. Nakamura and M. Yamaguchi, arXiv:hep-ph/0604132.

[5] M. Dine, R. Kitano, A. Morisse and Y. Shirman, Phys. Rev. D 73, 123518 (2006).

[6] M. Endo, K. Hamaguchi and F. Takahashi, arXiv:hep-ph/0605091.

[7] M. Dine, A. E. Nelson and Y. Shirman, Phys. Rev. D 51 (1995) 1362; M. Dine, A. E. Nelson, Y. Nir and Y. Shirman, Phys. Rev. D 53 (1996) 2658; For a review, see, for example, G. F. Giudice and R. Rattazzi, Phys. Rep. 322 (1999) 419, and references therein.

[8] L. Randall and R. Sundrum, Nucl. Phys. B 557, 79 (1999);

G. F. Giudice, M. A. Luty, H. Murayama and R. Rattazzi, JHEP 9812, 027 (1998);

J. A. Bagger, T. Moroi and E. Poppitz, JHEP 0004, 009 (2000).

[9] M. Ibe, Y. Shinbara and T. T. Yanagida, arXiv:hep-ph/0605252.

[10] E. Witten, Nucl. Phys. B 188, 513 (1981).

[11] I. Affleck, M. Dine and N. Seiberg, Phys. Rev. Lett. 52, 1677 (1984); Nucl. Phys. B 256, 557 (1985).

[12] M. Dine and A. E. Nelson, Phys. Rev. D 48, 1277 (1993).

[13] M. Dine, A. E. Nelson and Y. Shirman, in [7].

[14] K. I. Izawa and T. Yanagida, Prog. Theor. Phys. 95, 829 (1996)

[15] K. A. Intriligator and S. D. Thomas, Nucl. Phys. B 473, 121 (1996) 
[16] R. Allahverdi, K. Enqvist and A. Mazumdar, Phys. Rev. D 65, 103519 (2002).

[17] G. Lazarides and C. Panagiotakopoulos, Phys. Rev. D 52, 559 (1995).

[18] M. Kawasaki, K. Kohri and T. Moroi, Phys. Lett. B 625, 7 (2005); Phys. Rev. D 71, $083502(2005)$.

[19] K. Kohri, T. Moroi and A. Yotsuyanagi, Phys. Rev. D 73, 123511 (2006) [arXiv:hep-ph/0507245].

[20] K. Jedamzik, arXiv:hep-ph/0604251.

[21] T. Moroi, H. Murayama and M. Yamaguchi, Phys. Lett. B 303, 289 (1993).

[22] H. Ooguri and Y. Ookouchi, arXiv:hep-th/0606061.

[23] I. Affleck, M. Dine and N. Seiberg, Phys. Lett. B 137, 187 (1984); Phys. Lett. B 140, 59 (1984).

[24] D. H. Lyth and E. D. Stewart, Phys. Rev. D 53, 1784 (1996);

M. Kawasaki and F. Takahashi, Phys. Lett. B 618, 1 (2005);

see also M. Endo and F. Takahashi, arXiv:hep-ph/0606075.

[25] M. Bolz, A. Brandenburg and W. Buchmuller, Nucl. Phys. B 606, 518 (2001).

[26] D. N. Spergel et al., arXiv:astro-ph/0603449.

[27] M. Bastero-Gil, S. F. King and Q. Shafi, arXiv:hep-ph/0604198.

[28] M. Endo, M. Kawasaki and T. Moroi, Phys. Lett. B 569, 73 (2003).

[29] G. R. Dvali, Q. Shafi and R. K. Schaefer, in Ref. [2].

[30] T. Watari and T. Yanagida, Phys. Lett. B 589, 71 (2004).

[31] G. N. Felder, J. Garcia-Bellido, P. B. Greene, L. Kofman, A. D. Linde and I. Tkachev, Phys. Rev. Lett. 87, 011601 (2001);

G. N. Felder, L. Kofman and A. D. Linde, Phys. Rev. D 64, 123517 (2001).

[32] T. Asaka, W. Buchmuller and L. Covi, Phys. Lett. B 510, 271 (2001)

[33] E. J. Copeland, S. Pascoli and A. Rajantie, Phys. Rev. D 65, 103517 (2002).

[34] A. D. Linde, Phys. Lett. B 129, 177 (1983). 
[35] M. Kawasaki, M. Yamaguchi and T. Yanagida, Phys. Rev. Lett. 85, 3572 (2000); Phys. Rev. D 63, 103514 (2001).

[36] L. O'Raifeartaigh, Nucl. Phys. B 96, 331 (1975).

[37] M. Viel, J. Lesgourgues, M. G. Haehnelt, S. Matarrese and A. Riotto, Phys. Rev. D 71, 063534 (2005).

[38] M. Fukugita and T. Yanagida, Phys. Rev. D 42, 1285 (1990); see, for a review, W. Buchmuller, R. D. Peccei and T. Yanagida, Ann. Rev. Nucl. Part. Sci. 55, 311 (2005).

[39] K. Kumekawa, T. Moroi and T. Yanagida, Prog. Theor. Phys. 92 (1994) 437;

G. Lazarides, Springer Tracts Mod. Phys. 163 (2000) 227 and references therein;

G. F. Giudice, M. Peloso, A. Riotto and I. Tkachev, JHEP 9908 (1999) 014;

T. Asaka, K. Hamaguchi, M. Kawasaki and T. Yanagida, Phys. Lett. B 464 (1999)

12; Phys. Rev. D 61 (2000) 083512;

M. Kawasaki, M. Yamaguchi and T. Yanagida, Phys. Rev. D 63 (2001) 103514. 